\title{
Molecular simulations: past, present, and future (a Topical Issue in EPJB)
}

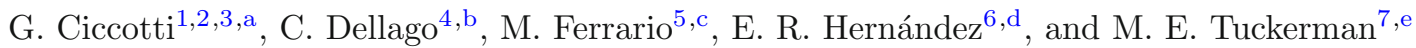 \\ 1 Department of Physics, University of Rome "La Sapienza", Rome, Italy \\ 2 AIC-CNR, Rome, Italy \\ 3 School of Physics, University College Dublin, Dublin, Ireland \\ 4 Faculty of Physics, University of Vienna, Vienna, Austria \\ ${ }^{5}$ Dipartimento di Scienze Fisiche, Informatiche e Matematiche, Università di Modena e Reggio Emilia, Modena, Italy \\ ${ }^{6}$ Instituto de Ciencia de Materiales de Madrid (ICMM-CSIC), Madrid, Spain \\ 7 Department of Chemistry and Courant Institute of Mathematical Sciences, New York University, New York, USA
}

Published online 5 January 2022

(C) The Author(s), under exclusive licence to EDP Sciences, SIF and Springer-Verlag GmbH Germany, part of Springer Nature 2021, corrected publication 2022

\section{Introduction: molecular simulation beyond the nutshell}

Molecular simulation (MS) could be concisely described as computational statistical mechanics [1], i.e., numerical methods combined with computer power to address physico-chemical problems arising in condensed matter systems. Given the laws governing the interactions between the atoms or molecules in the system (the model), one would use MS to quantify a variety of system properties as predicted by the model, such as thermal averages, transport coefficients, and perhaps even discover unforeseen microscopic processes in the system, such as reaction mechanisms, that no amount of educated guessing could have anticipated without the detailed microscopic view that MS offers. As we will discuss below, but even more in the articles published within this Special Issue (SI), such a nutshell description, while not wholly inaccurate, fails to do justice to the variety and richness of MS techniques currently in use in the scientific community.

One of the aims of this Special Issue is to provide a general view of how MS is growing, complementing the nutshell description given above. This SI aims to illustrate the most active directions of current research. Indeed, the field of fundamental computer simulations of matter is as active as it ever was, as will become apparent. We will be covering the fruitful interaction of atomistic simulations and Machine Learning, one of

\footnotetext{
${ }^{a}$ e-mail: giovanni.ciccotti@roma1.infn.it

b e-mail: christoph.dellago@univie.ac.at

c e-mail: ferrario@unimore.it

d e-mail: eduardo.hernandez@csic.es (corresponding author)

ee-mail: mark.tuckerman@nyu.edu
}

the most interesting developments to have taken place in the field in recent years. Progress in the sampling of rare events and non-equilibrium processes will also be discussed; we will delve into the most recent methods for integrating the equations of motion and addressing quantum dynamics, and new ideas on how to extend the applicability of computer simulations beyond the realm of atomistic descriptions of condensed matter by means of coarse-graining or continuum approaches will be explored. Finally, we also hope to provide some pointers for directions in which MS may evolve in the future.

To better appreciate where we are, and especially where we may be going from here, it is appropriate to look back and consider where we have come from. We do not aim to provide here a detailed historical perspective; that would be hardly appropriate in a preface article (readers interested in the details of the history of MS should consult Ref. [2]). Rather, we just want to remark on a few key landmarks that have contributed to give shape to what we now know as molecular simulation. We do so in Sect. 2. Then, in Sect. 3, we introduce the topics that are covered in this SI, put them in the context of the present state of the art, and discuss how they could contribute to the future evolution of MS.

\section{Molecular simulation: a brief history}

Computer simulations applied to condensed matter systems began in the 1950s. Two of the corner stones of molecular simulations were introduced then, namely the Monte Carlo (MC) sampling technique [3] and the molecular dynamics (MD) method [4]. That the two techniques appeared almost simultaneously at this 
time is no coincidence. During the previous decade, electronic computing machines had been developed to address many of the computational demands going beyond direct human capabilities that had to be confronted in connection with the war effort, and in particular the Manhattan project and the breaking of the Enigma code. A decade later, improved computers were still employed at several US national labs, primarily for military purposes, but with the war over, the high cost of such machines made it desirable to search for new civilian areas in which their capabilities could be exploited. It was thus that scientific applications began to take shape.

The basic idea behind $\mathrm{MC}$ is to estimate the average value of a system property by sampling over a collection of configurations generated at random from the probability distribution corresponding to the appropriate statistical mechanics ensemble (e.g., the canonical ensemble for systems at constant temperature); it is a numerical realisation of Gibbs's program. The idea behind MD is even more intuitive: simply, solve Newton's equations of motion for the classical particles in the system; in other words, it is the numerical realisation of Boltzmann's dream. Besides providing time averages of system properties (which in the limit of sufficiently long trajectories should approximately coincide with the configuration averages provided by $\mathrm{MC}$ ), MD allows one to monitor the time evolution of the system, thus giving access to what are commonly known as dynamical properties, in particular transport.

From today's perspective, MC and MD may seem like trivial, obvious procedures, but they were not so at the time. Indeed, it took a certain change of perspective to consider that numerical techniques and computer power could be combined to address models of matter for which, in spite of their small size and simplicity (e.g., 32 hard spheres [4]), the equations of statistical mechanics were analytically intractable. It was not simply a question of original thinking; simulation took some time to be accepted by a scientific community brought up in the belief that the job of a scientist was to discover the laws of nature, not necessarily to solve the resulting equations. However, the seed had been planted, and little by little, it started growing.

During the following decade, computers grew in power and speed, and also became more widely available to researchers both in the US and in Europe. In 1964, Rahman [5] published the first simulation results on a realistic model system, an MD simulation of liquid Argon. Previous MD simulations of Alder and Wainright [4] were used to simulate systems consisting of hard spheres, a discontinuous potential that counted more as a generic model than as a realistic one. The work of Rahman showed that MD simulations were indeed possible with smooth potentials (he used a Lennard-Jones pair-wise additive potential to account for the interactions between Ar atoms).

Basic algorithms and their improvements were essential, particularly in an age in which computers were still comparatively few and had limited speed and memory. Verlet [6] proposed a stable numerical integration scheme that carries his name, still very much in use today. He also introduced a time-saving algorithm, the Verlet neighbour list.

Gradually, an increasing number of scientific publications demonstrated the power of MS to help interpret experiments, and sometimes even predict their results. A remarkable step forward in this respect was the work of Rahman and Stillinger [7], who reported for the first time in 1971 an MD study on a model of liquid water, a system composed of molecules, not just atoms. This work was a significant achievement: it demonstrated that the structure of liquid water consisted of a random network of hydrogen bonds, having no structural resemblance to its solid phases; it also showed that the diffusion of water molecules took place continuously, and not via a hopping mechanism, as was widely assumed at the time. Beyond that, this work contributed more than any other published up to then to underline the message that MS was capable of greatly contributing to the advancement of science. Indeed, from today's perspective, the importance of the work of Rahman and Stillinger lies in the fact that it inspired the scientific community to think that, if it was possible to simulate water, which is after all the medium in which the chemistry of life takes place, why not simulate also biomolecules themselves? Although many difficulties had to be addressed first, the goal was nevertheless clear, and a multinational group of scientists centred around Berendsen at $\mathrm{CECAM}^{1}$ began to work, as early as 1972, with this objective in mind. The first MD simulation of a simple protein was due to Karplus and collaborators, and appeared shortly after, in 1977 [8].

One of the technical issues to be addressed was how to cope with the different time-scales in molecular systems, where vibrations and bond-bendings occur over a time-scale of a few femtoseconds $\left(10^{-15} \mathrm{~s}\right)$, while conformational changes can take place over macroscopic time-scales. The constraint method [9] was introduced to freeze out fast degrees of freedom, thus allowing the use of longer time-steps and consequently speeding up the simulations. The constraint method was to find important applications later, in the study of rare events [10] and in the combination of molecular dynamics with electronic structure calculations [11]. The efforts started then by many researchers on the simulation of biomolecules were eventually to lead to the award of the Nobel prize for Chemistry to Warshel, Levitt, and Karplus in 2013 for their contributions to computer simulations in biochemistry. Somewhat later, symplectic, time-reversible multiple time-scale (MTS) integration algorithms were introduced [12], which allowed different force components, such as bonded, short-range non-bonded, and long-range forces, acting on different time-scales to be integrated with an appropriate timestep. MTS integration allowed the largest time step, in biomolecular simulations to be increased to roughly 5$10 \mathrm{fs}$ while retaining the full physics built into modern atomistic force fields. As the largest time-step is asso-

\footnotetext{
1 Centre Européen de Calcul Atomique et Moléculaire.
} 
ciated with the computationally most expensive forces, the gains in efficiency were on a par with or somewhat better than those obtained using constraints. MTS algorithms, along with variable transformations, were key to providing a molecular dynamics-based approach for incorporating nuclear quantum effects via Feynman path integrals [13].

More or less in parallel with the development of models and techniques required to address biochemical systems, efforts were being made to extend the applicability of MS to other fields. One example of this was the case of quantum many-body systems: techniques to address quantum systems, such as variational Monte Carlo, had been developed during the 1960s, but the computer power required to apply them to truly manybody systems was simply not available at the time. It was not until the late 1970 s and 1980 s that the work of Ceperley and Alder to address such systems as the electron gas [14] and hydrogen at high pressures [15] became computationally feasible. Another example was the study of liquid crystals. The chemical complexity of such systems, usually colloids or complex organic molecules, gave many the impression that these systems would not be amenable to simulation. However, the work of Frenkel and collaborators [16,17] demonstrated that the phase dynamics of liquid crystals was controlled by their shape rather than by their chemical interactions.

Another landmark in our leapfrog (pun intended) path along the history of MS took place in 1980. In this year, Andersen published a paper [18] which was to have wide repercussion. In short, Andersen described how to extend MD to enable it to sample the isoenthalpic (constant pressure) ensemble. The standard MD algorithm samples the microcanonical (constant energy) ensemble, since Newton's equations of motion conserve the total energy, and it was not immediately apparent how could one modify MD to sample other, more experimentally relevant conditions. Andersen's solution to achieving constant-pressure MD sampling was to extend the dynamical variables of the system to include the volume; in other words, the volume became a new dynamical variable, with a fictitious mass and a velocity (or momentum) associated with it. With a term $P_{\text {ext }} V$ added to the potential energy, where $P_{\text {ext }}$ is the external pressure and $V$ the volume, this method, commonly known as Andersen's barostat, can be seen to sample the isoenthalpic ensemble at pressure $P=P_{\text {ext }}$. Shortly after, Parrinello and Rahman [19,20] showed how the scheme could be generalised to include shape as well as volume fluctuations. This was an important generalisation, as it made possible to consider such problems as crystallisation and solid-solid-phase transitions.

Following Andersen's extension of MD to sample the isoenthalpic ensemble, the question arose as to whether it was possible to find a similar scheme allowing MD to sample the canonical ensemble. Nosé [21,22], following in the steps of Andersen, incorporated a new dynamical variable coupling the kinetic energy of the atoms to the external (bath) temperature, in such a way that the resulting dynamics can be shown to sample the desired ensemble. This is now known as the Nosé-Hoover thermostat, as it is most frequently used in a modification due to Hoover [23]. Andersen's idea of extending the system's dynamical variables to include fictitious ones such as a barostat, or Nosé's thermostat, is not only ingenious; it is actually quite remarkable that a single additional degree of freedom can effectively play the role of an entire pressure or temperature reservoir.

In 1985, Car and Parrinello [11] published a paper in Physical Review Letters which was to take the simulation community by storm. Their paper offered a scheme to combine MD with the direct calculation of the electronic structure by means of density functional theory (DFT). Effectively, this obviated the need to rely on a potential model: energy, forces, and stress could be obtained directly from the electronic structure calculation. Bonds could be formed or broken during the simulation. The idea was to combine the "real" dynamics of the ions with a fictitious dynamics of the electronic degrees of freedom, designed in such a way that it would remain adiabatically separated from the slower dynamics of the ions, while at the same time keeping close to the electronic ground state.

The Car-Parrinello method was indeed a very important breakthrough. For one thing, it showed for the first time that it was possible to combine finite temperature simulations and ground-state electronic structure calculations, something that up to that point, most scientists would have regarded as far too computationally intensive to be practical. On the other hand, it also served as a bridge between scientific communities: the simulation community, that generally had a statistical mechanics background, and the solid-state physics and quantum chemistry communities, working on electronic structure calculations at zero temperature.

During the 1980s and 1990s, MS studies in condensed matter became more common, thanks in part to earlier successes and also to ever growing computer power and its wider availability. However, as is always the case in science, previous advances had only brought nearer and made more evident the significant challenges that still lied ahead. One of these challenges was the study of phase equilibria. In spite of the dramatic increase in computer power experienced since the early days of MS, this problem was still regarded as largely out of reach, due to the apparent need to explicitly include the interface. However, Panagiotopoulos [24] devised an MC algorithm, known as Gibbs ensemble Monte Carlo, that required only the simulation of the involved phases, and bypassed any need to consider the interface.

Another challenge, perhaps the most fundamental one, is the question of time-scales and rare events. The long-time dynamics of many processes (e.g., protein folding) is dominated by the presence of free energy barriers that separate long-lived configurations or states of the system. Conventional MS techniques are particularly adept at exploring the short-time-scale dynamics (MD) or the more probable regions of configuration space (MC), but they are not efficient when it comes to rare events. New algorithms and theoretical methods were needed, and began to appear at this time, 
such as the Blue Moon ensemble [10], minimum-energy path methods such as the Nudged-Elastic Band [25] and the String [26] method, dynamical algorithms, such as hyper-MD [27] or metadynamics [28]. Path sampling methods were also developed at this time [29], eventually leading to the development of transition-path theory [30]. Another possible avenue was recently opened by applying some important mathematical results on Markov processes to the study of metastability [31].

Beyond the question of spanning the multiple timescales present in many physico-chemical processes in condensed matter systems, there is also the question of addressing different length scales. Certain problems, such as fracture, catalysis, or biochemistry, require a description of the system that encompasses the atomistic level, where chemical bonds are formed and broken, all the way up to the mesoscopic level and beyond. Clearly, these different length scales cannot be treated at the same level of detail, and it becomes necessary to seamlessly match different levels of description in a physically consistent way. Incidentally, this was a problem already confronted by early simulations of biomolecules; indeed, the work of Warshel and Levitt [32] to address enzymatic reactions involved combining a quantum-mechanical description of the fragments where chemical reactivity requires it with a molecular mechanics description for the rest. This work was to eventually lead to their sharing of the 2013 Chemistry Nobel prize with Karplus (see above).

To conclude this bird's eye view of the history of MS, it is perhaps appropriate to remark that nowadays MS has clearly established itself as a key scientific instrument, capable of aiding in the interpretation of experimental results, of suggesting new experiments, and more and more frequently even predicting their results. That this is so is demonstrated by the growing number of publications in which both experiments and simulation tools are used in tandem. However, in spite of the remarkable advances detailed above (and many others that we could not do justice to), MS is still subject to numerous limitations, and, as will be discussed in the following section, the simulation community is constantly searching for new ways in which to overcome them. This search is what will drive the future evolution of the field.

\section{Molecular simulation: the best is yet to come}

While molecular simulation has evolved at breathtaking speed from its beginnings in the 1950s, driven by the rapid increase of raw computing power, the invention of clever algorithms, and the development of efficient and user-friendly software packages, it is still some distance away from the grand, but perhaps a little too optimistic vision of making it possible to understand and accurately predict emergent phenomena all the way from the atomistic to the macroscopic scale. For instance, the precise prediction of phase diagrams of materials from first principles is often out of reach of current capabilities and so is the simulation of many biomolecular processes. While the growth in computational power continuously pushes the boundaries of what is feasible, we are currently witnessing several promising algorithmic and conceptual developments that have the potential to expand the scope of molecular simulation and are the focus of this special issue.

\subsection{Machine learning}

While, as famously remarked by Dirac, the basic natural laws that determine the interactions of atoms and molecules are known, often their application is computationally extremely expensive. In the early days of computer simulations, this problem was circumvented by postulating the functional form of interatomic interactions and adapting parameters, so that the simulations reproduce selected experimental data or results of high-accuracy quantum mechanical calculations. This so-called force-field approach did not only provide numerous insights into the generic mechanism of many physical processes, but is still the basis for most atomistic/molecular simulations of complex systems in biology and materials science. In parallel, approximate quantum mechanical methods such as coupled cluster theory and density functional theory were developed and increasingly used in molecular simulations. Such calculations, however, are computationally very demanding, severely limiting accessible system sizes, and simulation times. Recently, data-driven machine learning approaches have been shown to provide a convenient way to circumvent this problem and combine the advantages of inexpensive force fields with the accuracy of ab initio methods.

The main idea in the application of machine learning to the calculation of energies and forces in molecular simulations is to use high-precision reference data obtained from costly electronic structure calculations to parameterize inter-atomic potentials represented by flexible machine learning models such as neural networks [33] or kernel-based approaches [34]. Once properly trained, machine learning models accurately interpolate between the reference data, producing force fields that deliver the accuracy of ab initio calculations at a fraction of their cost. The development of such ML force fields, which can easily model also chemical reactivity, relies on the availability of large sets of training data that are usually constructed iteratively, but recently on-the-fly approaches have been put forward to generate reference data only when needed $[35,36]$. Since with a sufficiently large set of training data, the accuracy of the machine learned model will be limited mainly by the quality of the reference data, machine learning-based simulations will expose any deficiencies of the computational methods used to produce the reference data, providing a further drive for the development of more accurate quantum mechanical methods. Due to their computational efficiency, machine learn- 
ing methods make it more and more possible to apply ab initio methods to large systems and truly go all the way from the atomistic description to the calculation of macroscopic properties, eventually completing the unification of the statistical mechanics and electronic structure communities initiated by the advent of the Car-Parrinello method [11] in the mid-1980s.

The significance of machine learning in molecular simulation, however, goes far beyond the development of computationally inexpensive yet accurate force fields. Enormous amounts of data are produced every day by molecular dynamics simulations running on highperformance computers around the world and one of the big challenges is to make sense of this data rather than to generate them. In particular, clustering, dimensionality reduction and pattern recognition, and classical methods of machine learning can be applied to discover hidden patterns and regularities in the output of large-scale molecular simulations. Such machine learning approaches help to identify the (hopefully) few degrees of freedom that capture the essential features of the process under study and that can be used to construct coarse-grained lower dimensional models amenable to human understanding [37,38]. Furthermore, generative models based on learned mappings promise to provide a way to sample the equilibrium distribution efficiently, bypassing the statistical correlations that often hamper molecular dynamics and Monte Carlo simulation [39,40]. Finally, let us mention that the relation between molecular simulation and machine learning is not necessarily a oneway street. Techniques developed to explore the rugged potential energy surface of complex molecular systems may turn out to be helpful in training deep learning models by viewing them as interacting particle systems [41].

\subsection{Rare events and enhanced sampling}

The advances in machine learning approaches mentioned in the previous section are also likely to have an impact on the simulation of processes dominated by rare events, for instance phase transitions, chemical reactions, or biomolecular rearrangements. Such processes are characterized by widely separated timescales arising from narrow energetic or entropic bottlenecks that the system needs to cross when transitioning between long-lived states. The resulting long waiting times represent a significant computational challenge and often preclude the straightforward simulation of rare but important barrier crossing events. While in the past 50 years, big advances have been made in the development of simulation methods to circumvent this problem [42], devising more efficient methods for the sampling and, perhaps even more importantly, the analysis of rare events is still a very active area of research, as is demonstrated by the related contributions included in this special issue.

Since rare events are caused by rarely visited barriers, one idea to solve the rare event problem in molecular simulations consists in artificially enhancing the probability to sample these configuration space regions by applying a suitable bias or constraint. In the equilibrium case, the effect of the bias can then be removed easily by reweighting. This general biasing strategy has been widely and very successfully applied in many forms, either using static biases like in umbrella sampling [43] or building up the bias function dynamically as in metadynamics [44], flat histogram sampling [45], or the nested sampling methods discussed in this special issue [77]. As a result of such calculations, one typically obtains free energy profiles along selected degrees of freedom, which can be complemented with dynamical information obtained from unbiased dynamical trajectories initiated in barrier regions to obtain information on the kinetics of the process under study.

In general, biasing schemes work well, if one has a good idea of the collective variables governing the evolution of the system. In this case, free energy barriers correspond to true dynamical bottlenecks traversed by the system. Particularly for complex disordered systems, however, characterizing the dynamics in terms of a few relevant collective variables is a highly non-trivial problem and while corresponding free energy landscapes might look meaningful, they can be deceptive in terms of kinetics and mechanism. Path-based methods have been devised exactly to avoid this problem and to study rare events without prior mechanistic knowledge. Some of these approaches, including the nudged-elastic band method [25] or the string method [26], are based on artificial pathways that connect stable states in an unbiased way, but do not obey the underlying dynamical rules. Other methods, such as transition-path sampling $[46,47]$, forward flux sampling $[48,49]$, and milestoning [50], employ dynamical trajectories that embody the true time evolution of the system. Here, the bias that ensures reactivity is applied at the trajectory level without affecting the natural dynamics the system follows along each individual trajectory.

While the ability to sample rare events is crucial for the investigation of many condensed matter processes, it is only the first step towards mechanistic understanding. Further analysis of the generated pathways is necessary to identify important degrees of freedom that capture the essential features and to construct meaningful low-dimensional models. The probabilistic framework of transition-path theory [51] and of Markov state models [52] offers the theoretical underpinning for this task, which will become more and more important as the amount of generated data continues to increase at a rapid pace. Machine learning approaches are increasingly used for the sampling and analysis of rare event trajectories as exemplified in the application of the transition manifold analysis to amyloid fibrillation discussed in the contribution of Bittracher et al. [76]. Other exciting developments in this area include the application of maximum entropy and maximum caliber principles to incorporate experimentally known rate constants into trajectory-based simulations presented in the article of Bolhuis et al. [78]. 


\subsection{Transport and non-equilibrium molecular dynamics}

On a macroscopic level, the transport of conserved quantities like mass, charge, momentum, and energy in response to weak external perturbations is described in terms of phenomenological equations, such as Fourier's law for heat conductions or Fick's law for diffusion, in which all microscopic details are condensed into the values of some material specific transport coefficients. The perhaps most direct method to compute these coefficients is to carry out non-equilibrium molecular dynamics simulation and monitor the currents induced by external fields or gradients [53]. Alternatively, the connection to the microscopic dynamics of the system is provided by the Green-Kubo theory of linear response $[54,55]$. In this framework, transport coefficients such as the diffusivity, the shear viscosity, and the thermal conductivity are expressed as integrals over the equilibrium time autocorrelation function of the appropriate fluxes. The correlation functions can be computed in equilibrium MD simulations, and this approach has been used extensively to investigate transport processes in many systems ranging from solids to liquids and complex soft materials. Often, however, autocorrelation functions decays very slowly, particularly in solids but also in dilute gases, such that excessively long simulation times are needed to converge the results. As discussed in the contribution by Grasselli and Baroni [80] and demonstrated using heat transport in insulators and charge transport in ionic conductors as examples, this problem can be significantly alleviated by exploiting the gauge invariance of transport coefficients combined with a new spectral analysis of the fluxes.

A particular challenge in the field of transport lies in the simulation of phoretic phenomena such as thermophoresis and diffusiophoresis, in which the motion of colloidal particles is driven by gradients in the surrounding fluid [56]. The magnitude of the phoretic motion and sometimes even its direction may depend in unexpected ways on parameters such as salinity and $\mathrm{pH}$ of the solvent, temperature, solute concentration, specific surface coatings, and molecular weight. Phoretic processes, however, are difficult to model with computer simulations due to the disparate time and length scales that arise, because the colloids are much bigger than the solvent molecules. Moreover, computer simulations of this complex phenomenon need to both include thermal fluctuations and reproduce hydrodynamic flow. The challenges arising in the computer simulation of phoretic transport as well as recent progress in this area are the subject of the article by Ramírez-Hinestrosa and Frenkel included in this special issue [79].

While in the case of weak perturbations, transport properties are related to equilibrium fluctuations, these relations break down for large forces that drive the system far from equilibrium. In this case, which is particularly relevant at the nanoscale, transport properties are dominated by large fluctuations and non-linear behavior arises. The statistics of non-equilibrium fluctuations can be analysed in the framework of stochastic thermo- dynamics [57] and large deviation theory [58] provides the mathematical formalism to determine higher order transport coefficients in numerical simulations of nonequilibrium steady states, as discussed in the contribution by Limmer [81]. The full non-linear response of a system to an external perturbation is also considered in the dynamical non-equilibrium approach (D-NEMD) method often applied to study biomolecular systems and reviewed in the article of Oliveira et al. [89].

\subsection{Adiabatic separation, dynamics, and statistics}

Time-scale separation in the evolution of complex systems is a well known phenomenon in theoretical physics, and has been used many times in the past, in quantum and classical dynamics to justify successful approximations in the solution of relevant questions. Possibly, the most widespread use of this phenomenon is with the Born-Oppenheimer (BO) approximation, in which one assumes that the electrons stay in their ground state (parametrically dependent on the nuclear coordinates), while the nuclei slowly move in the (mean) field produced by the electrons around them. It is difficult to underestimate the importance of the $\mathrm{BO}$ approximation, since a large part of condensed matter physics is based on it. Beyond that, however, the adiabatic approximation is used also in the solution of the dynamics of classical many-body systems where it finds an appropriate expression close in spirit to the BO one. Very often, in partial treatments, the use of the approximation is limited to the solution of the problem associated with the simplified system generated by the adiabatic separation without tackling the solution of the whole coupled problem. In molecular simulation, instead, the association of computer power and smart algorithms has permitted to address the problem in its entirety, solving on the fly the "evolution" (in the quantum case, the calculation of the ground state) of the fast variables to drive that of the slow variables .

Of course, the invention of suitable algorithms is essential and never a trivial job. However, as we have already recalled, in the 80s, Car and Parrinello gave the good way to solve this problem and created what is normally called Car-Parrinello molecular dynamics [11]. On the footpath of this success, Sprik, a few years later, suggested a smart way to solve the dynamics of polarizable models which are built in a way that can be read as two set of adiabatically separated variables [59]. Another 10 years elapsed before, first, Röthlisberger and, then, Vanden Eijnden and Maragliano produced an algorithm to sample the mean force in a statistical system adding to it some extra-variables adiabatically separated by the natural ones [60]. All in all, a magnificent demonstration of the power of the method is to solve complex dynamical situation. This line of research is far from being abandoned, and recently, there have been at least a couple of advances worth recording. This is why, in this collection, you will find a paper on the exact adiabatic separation of real and virtual degrees of freedom by exploiting the motion of zero-mass par- 
ticles constrained to move on an hypersurface of the real variables space [82] and another on an attempt to improve stability and performances of the original Car-Parrinello approach by introducing a new extended Lagrangian in place of the original one [83].

\subsection{Integrators: deterministic and stochastic}

The molecular dynamics approach is based on the solution of Newton's equations of motion, which, for a system of $N$ particles, constitute a set of $3 N$ second-order ordinary differential equations. For any realistic system, the forces in these equations are of such complexity that the equations cannot be solved analytically, and therefore, a numerical integrator or solver must be used. Numerical integrators for Newton's equations must operate on both a formal and a practical level. On a formal level, an integrator should preserve, to the greatest extent possible, the underlying physics of the equations of motion. Since Newton's equations are equivalent to Hamilton's equations of motion, the integrator should preserve their underlying symplectic structure and, thereby, satisfy Liouville's theorem of classical statistical mechanics, so that the energyconservation error is bounded and phase-space volume is preserved. On a practical level, in a molecular dynamics simulation, whatever tricks are employed to speed up the calculation of the forces, ultimately, it is the force calculation that is the most time-consuming part of the simulation. Therefore, the time-scale that can be accessed in a molecular dynamics simulation will be limited by the number of force evaluations needed to reach that time-scale. The accuracy of an integrator determines the number of force evaluations needed and, therefore, controls the efficiency of the simulation. The same practical considerations must be taken into account in designing integrators for stochastic simulations based on the Langevin or generalised Langevin equation or for deterministic thermostats and barostats for sampling isothermal and/or isobaric ensembles.

The MTS integrators mentioned in Sect. 2 represent a perfect marriage of algorithms that preserve the formal structure of the underlying equations while attempting to enhance the efficiency of a simulation $[12,13]$. The formal aspects of an MTS algorithm are captured via the use of a Trotter factorized classical propagator derived from the Liouville operator, a formalism that has proved extremely powerful in designing numerical solvers for both deterministic and stochastic equations of motion. Unfortunately, when forces are decomposed by time-scale and the decomposition is in an MTS algorithm, numerical "resonances" emerge, in which frequencies associated with the fast forces limit the time steps that can be chosen for the slower force components. This is why the largest time-step is fundamentally limited to the 5-10 fs range, even though temporal variations in long-range forces should allow a much longer time-step to be employed. Solving the resonance problem has been a major challenge in the design of efficient and rigorous molecular dynamics integrators. One of the contributions [85] will discuss several strategies for overcoming these resonances and allowing use of very large time-steps approaching 100 fs to be employed within an MTS framework. These will include isokinetic and regulated dynamics schemes for sampling commonly used ensembles and computing equilibrium properties and methods derived from the generalised Langevin equation for generating dynamical observables. In a complementary function, we also have a contribution [84] on how to optimise the sampling by an appropriate choice of the damping coefficients in Langevin dynamics.

Beyond the issues highlighted above, new challenges are already arising in the design of numerical solvers. As new software and hardware paradigms continue to transform the landscape of molecular dynamics calculations, integrators, particularly MTS integrators, will need to keep up with these advances, which will present interesting challenges going forward. For example, the use of accelerators such as graphical processing units (GPUs), used either as the principal computational engine or in conjunction with CPUs, changes the balance in computation time between different force components, which could affect the performance of MTS integrators. In addition, potential energy models based on machine learning of quantum chemical calculations do not readily present ways to be decomposed based on time-scales. Therefore, new strategies for working with such potential models within the MTS framework will be needed, as the computational overhead of machine learning potentials is still significant compared to commonly used empirical potential models. Advances such as these will inevitably drive the design of next-generation algorithms for molecular dynamics simulations.

\subsection{Quantum dynamics}

For classical many-body systems, we can rely on Molecular Dynamics or Monte Carlo methods to study the model behavior. The dimensionality is important, but at the same time, it is in no way limiting the possibility to study in depth the statistical mechanics of our models. Essentially, there are no restrictions on the classical models that can be treated in this manner [61]. Also, in the Born-Oppenheimer approximation, assuming no breaking of the adiabatic separation hypothesis, the interactions between the particles can be obtained on the flight from quantum mechanics (normally timeindependent density functional theory) without ruining the validity of the scheme [62].

The situation is still palatable with time-independent statistical properties of bosonic quantum systems thanks to the path-integral formulation of quantum statistical mechanics in imaginary time by Feynman and its (partial) isomorphism with a model of classical polymers [63]. Unfortunately, for the dynamics of quantum systems, in particular fermionic, the situation is not as good as in the classical case. From the computational point of view, which is our focus here, the complica- 
tion arises from the fact that quantum mechanics is a theory that is formulated in terms of operators (to us, matrices, but that does not improve the situation) that generally do not commute. This complication, for the dynamics and for fermionic systems, has dramatic consequences, since it makes construction and justification of algorithms for molecular simulation an extremely difficult task. It is a legitimate statement to say that, still at present, there is no simulation method for quantum dynamics that has power and applicability comparable to that of the techniques developed for classical systems $[64,65]$. Consequently, still now, the real challenge is to try to improve the existing quantum simulation methods (normally applicable only to systems of low dimensionality) or to invent new ones.

Molecular-level phenomena are often, intrinsically, of quantum mechanical character and involve tunneling, non-Born-Oppenheimer effects, and quantummechanical phase coherence. Many, sometimes ad-hoc, techniques have been developed and applied to study small molecular species or at the expense of the realism of the model when referring to complex molecular systems. Much hopes were put, e.g., in semiclassical methods [66]. However, in spite of the ingenious ideas and skilled algorithms used, the approach could not really afford the level needed for truly condensed matter applications. Therefore, these approaches fall somehow outside of the scope of our specific subject, the simulation of statistical aggregates of molecules, and will not find here an appropriate treatment.

It is worth recalling, though, the efforts devoted to solve quantum dynamics in condensed phases by approaches mixing quantum (for a small subsystem) and classical (for the large remaining part, the environment) dynamics. The starting point for these approaches, still much in use, is the celebrated surfacehopping method whose foundation though has remained essentially intuitive [67]. Attempts to put it on a more rigorous basis, be them originated by the approximation called quantum-classical Liouville dynamics or by the iterative linearised density matrix propagation scheme [68], have only in part succeeded, so that, until now, the progress has remained cumulative and even the fundamental question of the possibility of a consistent nonadiabatic quantum-classical statistical mechanics has been raised with some ground [69].

All in all, the situation remains open but a real breakthrough is not yet available or even in sight. Very recently, some more fundamental approaches devoted to discover new perspectives and algorithms to treat the full quantum dynamics problem in statistical mechanics systems have emerged. That is the main motivation for us to include a couple of papers characterizing this new promising trend: the so-called exact factorization approach aiming from the scratch to go beyond the Born-Oppenheimer approximation [86] and an evolution of the time-honored, but until now numerically unsuccessful, standard path-integral approach to quantum dynamics which has been recently successfully revived [87].

\subsection{New addressable complex phenomena}

The history of MS (see Sect. 2) is not only that of the tools and techniques that constitute its theoretical body; it is also the history of the problems that became tractable thanks to those techniques, and that motivated their development in the first place. Indeed, it is the drive to answer new questions concerning ever more complex phenomena that provides the main motivation for the continuing development of new theoretical tools and algorithms in MS. In the last section of this Topical Issue, we have collected contributions that aim to illustrate the kinds of systems that can be considered nowadays, the questions that can be asked (and answered) about them, and the methodological developments that make this possible.

It is obvious that biological systems continue to pose a great challenge to MS, making stringent requirements on both models and algorithms. Over the years, a number of methods have been put forward to speedup sampling of configuration space, such as transitionpath determination (nudged-elastic band [25] or string [26] methods), stochastic-Markov modelling, etc. The paper by Thomas and Roux [88] discusses how such methodologies apply and can be combined with datadriven Machine-Learning techniques in the context of biomolecules. How such systems respond to external perturbations (e.g., changing ambient conditions, electromagnetic fields, etc.) is also an important problem that can be addressed with the dynamical approach to non-equilibrium MD (D-NEMD), as discussed in the paper by Oliveira [89].

Coarse graining has been frequently used to reduce the complexity of modelled systems. The paper by Zhou and Glotzer [90] discusses a strategy for the inverse design of potentials for colloidal nanoparticles having a predefined ground state structure. In the paper by Cortes-Huerto et al. [91], the authors discuss how to cope with the boundary between two regions employing different levels of description, while Kidder and coworkers [92] focus on questions of design of coarse-grained models, with the requirement of reproducing energetic and entropic properties of the reference (atomistic) model. Finally, Menichetti et al. [93] introduce a metric to analyse and quantify the relative merits of different coarse grained representations of a given reference atomistic system.

An illustration of how MS can complement and aid experimental efforts is provided by the work of Bernetti and Bussi [95], who employ MS-generated biomolecular configurations to predict small-angle X-ray scattering (SAXS) spectra, an experimental tool used to probe the structure of biomolecules in solution.

Finally, we consider another problem of great fundamental and technological relevance: that of adsorption/desorption of liquids in nanoscaled pores and cavities, discussed in the paper by Giacomello [94]. Confinement at the nanoscale level affects wetting, evaporation, transport phenomena, etc. To harness the potential technological applications of nanoporous materi- 
als, a microscopic understanding of these phenomena is essential.

So far, we have concentrated our attention to problems which can be, if not solved completely, at least properly addressed by MS. Let us now try to exemplify something still waiting to be properly addressed. Nonequilibrium has been applied to cases in which time evolution can be followed by standard, stationary or even time-dependent molecular dynamics. Instead, how to deal with those in which the non-equilibrium time evolution of a system is hampered by the presence of an energy barrier is still a question that remains to be properly formulated. This surely represents an interesting not yet fully formalized situation in which nonequilibrium is inextricably coupled to rare event techniques. Something can be already done with selected methods, e.g., transition-path sampling [70,71] or forward flux sampling [48]. However, this is a more general new situation that, so far, has received limited attention.

\section{Content of the issue}

\subsection{Machine Learning (ML) meets $M D$}

\subsubsection{Potentials}

J. Behler, G. Csányi, Machine learning potentials for extended systems: a perspective [72]

J. Köfinger, G. Hummer, Empirical optimization of molecular simulation force fields by Bayesian inference $[73]$

\subsubsection{Enhanced Sampling}

M. Chen, Collective variable-based enhanced sampling and machine learning [74]

\subsection{Recent progress in rare events}

\subsubsection{Reaction Coordinates}

J. Rogal, Reaction coordinates in complex systems - a perspective [75]

\subsubsection{Markov State MC \& beyond}

A. Bittracher, J. Moschner, B. Koksch, R. Netz, C. Schütte, Exploring the locking stage of NFGAILS amyloid fibrillation via transition manifold analysis [76]

\subsubsection{Nested Sampling}

L. B. Pártay, G. Csányi, N. Bernstein, Nested sampling for materials [77]

\subsubsection{Max-Entropy to combine Exp \& Sim}

P. G. Bolhuis, Z. Faidon Brotzakis, M. Vendruscolo, A maximum caliber approach for continuum path ensembles [78]

\subsection{NEMD}

\subsubsection{Problems to be solved}

S. Ramírez-Hinestrosa, D. Frenkel, Challenges in modelling diffusiophoretic transport [79]

\subsubsection{Progress in transport by Kubo approach}

F. Grasselli, S. Baroni, Invariance principles in the theory and computation of transport coefficients [80]

\subsubsection{Transport \& Large deviations}

D. T. Limmer, C. Y. Gao, A. R. Poggioli, A large deviation theory perspective on nanoscale transport phenomena [81]

\subsection{Adiabatic separation, dynamics and statistics}

D. D. Girardier, A. Coretti, G. Ciccotti, S. Bonella, Mass-Zero constrained dynamics and statistics for the shell model in magnetic field [82]

A. M. N. Niklasson, Extended Lagrangian BornOppenheimer molecular dynamics: from density functional theory to charge relaxation models [83]

\subsection{Integrators, deterministic \& stochastic}

\subsubsection{General/Mathematical}

R. D. Skeel, C. Hartmann, Choice of damping coefficient in Langevin dynamics [84]

Resonance free multitime stepping

C. R. A. Abreu, M. E. Tuckerman, Multiple timescale molecular dynamics with very large time steps: avoidance of resonances [85]

\subsection{Quantum Dynamics}

\subsubsection{Exact factorization with trajectories}

F. Agostini, E. K. U. Gross, Ultrafast dynamics with the exact factorization [86]

Path Integral dynamics

S. C. Althorpe, Path-integral approximations to quantum dynamics [87] 


\subsection{New addressable complex phenomenologies}

\subsubsection{New trends in biological simulations}

T. Thomas, B. Roux, Tyrosine kinases: complex molecular systems challenging computational methodologies [88]

A. S. F. Oliveira, G. Ciccotti, S. Haider, A. J. Mulholland, Dynamical nonequilibrium molecular dynamics reveals the structural basis for allostery and signal propagation in biomolecular systems [89]

\subsubsection{Colloids \& active matter}

P. Zhou, S. C. Glotzer, Inverse design of isotropic pair potentials using digital alchemy with a generalized Fourier potential [90]

\subsubsection{Adaptive Resolution MD}

R. Cortes-Huerto, M. Praprotnik, K. Kremer, L. Delle Site, From adaptive resolution to molecular dynamics of open systems [91]

\subsubsection{Coarse grained dynamics}

K. M. Kidder, R. J. Szukalo, W. G. Noid, Energetic and entropic considerations for coarse-graining [92]

R. Menichetti, M. Giulini, R. Potestio, A journey through mapping space: characterising the statistical and metric properties of reduced representations of macromolecules [93]

\subsubsection{Cavitation and all that}

A. Giacomello, C. M. Casciola, Y. Grosu, S. Meloni, Liquid intrusion in and extrusion from non-wettable nanopores for technological applications [94]

\subsubsection{Developments in the study of RNA}

M. Bernetti, G. Bussi, Comparing state-of-the-art approaches to back-calculate SAXS spectra from atomistic molecular dynamics simulations [95].

Acknowledgements The authors wish to express their gratefulness for the assistance offered by Sandrine Karpe, Christiane Winter-Todorov and Isabelle Auffret-Babak during the preparation and publication of this article. The work of E. R. H. is supported by MICINN through grant No. PGC2018-096955-B-C44.

Data availability statement This manuscript has no associated data or the data will not be deposited. ôAuthors' comment: No data is deposited because this is strictly not a research article, but a preface article to a topical issue.]

\section{References}

1. M.E. Tuckerman, Statistical Mechanics: Theory and Molecular Simulation, Oxford Graduate Texts (Oxford University Press, Oxford, New York, 2010)

2. G. Battimelli, G. Ciccotti, P. Greco, Computer Meets Theoretical Physics (The new frontier of Molecular Simulation), The Frontiers Collection (Springer, Berlin, 2020)

3. N. Metropolis, A. W. Rosenbluth, M. N. Rosenbluth, A. H. Teller, and E. Teller, J. Chem. Phys. 21, 1087 (1953)

4. B. J. Alder and T. E. Wainright, J. Chem. Phys. 27, 1207 (1957)

5. A. Rahman, Phys. Rev. 136, A405 (1964)

6. L. Verlet, Phys. Rev. B 159, 6189 (1967)

7. A. Rahman, F.H. Stillinger, J. Chem. Phys 55, 3336 (1971)

8. J. A. McCammon, B. R. Gelin, and M. Karplus, Nature 267, 585 (1985)

9. J. P. Ryckaert, G. Ciccotti, and H. J. C. Berendsen, J. Comput. Phys. 23, 327 (1977)

10. E. A. Carter, G. Ciccotti, J. T. Hynes, and R. Kapral, Chem. Phys. Lett. 156, 472 (1989)

11. R. Car and M. Parrinello, Phys. Rev. Lett. 55, 2471 (1985)

12. M. Tuckerman, B. J. Berne, and G. J. Martyna, J. Chem. Phys. 97, 1990 (1992)

13. M. E. Tuckerman, B. J. Berne, G. J. Martyna, and M. L. Klein, J. Chem. Phys. 99, 2796 (1993)

14. D. M. Ceperley and B. J. Alder, Phys. Rev. Lett. 45, $566(1980)$

15. D. M. Ceperley and B. J. Alder, Phys. Rev. B 36, 2092 (1987)

16. D. Frenkel and J. P. McTague, Phys. Rev. Lett. 42, 1632 (1979)

17. D. Frenkel, B. M. Mulder, and J. P. McTague, Phys. Rev. Lett. 52, 287 (1984)

18. H. C. Andersen, J. Chem. Phys. 72, 2384 (1980)

19. M. Parrinello and A. Rahman, Phys. Rev. Lett. 45, 1196 (1980)

20. M. Parrinello and A. Rahman, J. Appl. Phys. 52, 7182 (1981)

21. S. Nosé, Mol. Phys. 52, 255 (1984a)

22. S. Nosé, J. Chem. Phys. 81, 511 (1984b)

23. W. G. Hoover, Phys. Rev. A 31, 1695 (1985)

24. A. Z. Panagiotopoulos, Mol. Phys. 61, 813 (1987)

25. H. Jónnson, G. Mills, K. W. Jacobsen (1998) In: B. J. Berne, G. Ciccotti, and D. F. Cocker (eds) Classical and Quantum Dynamics in Condensed Phase Simulations. World Scientific, Singapore, p. 385

26. W. E. Ren, and E. Vanden-Eijnden, Phys. Rev. B 66, $052301(2002)$

27. A. F. Voter, J. Chem. Phys. 106, 4665 (1997)

28. A. Laio and M. Parrinello, Proc. Natl. Acad. Sci. USA 99, $12562(2002 a)$

29. C. Dellago, P. G. Bolhuis, F. S. Csajka, and D. Chandler, J. Chem. Phys. 108, 1964 (1998)

30. W. E. Ren and E. Vanden-Eijnden, Ann. Rev. Phys. Chem. 61, 391 (2010)

31. C. Schütte and M. Sarich (eds) Metastability and Markov State Models in Molecular Dynamics (AMS, New York, 2013)

32. A. Warshel and M. Levitt, J. Mol. Biol. 103, 227 (1976) 
33. J. Behler, M. Parrinello, Phys. Rev. Lett. 98, 146401 (2007)

34. A.P. Bartók, M.C. Payne, R. Kondor, G. Csányi, Phys. Rev. Lett. 104, 136403 (2010)

35. Z. Li, J.R. Kermode, A. De Vita, Phys. Rev. Lett. 114, 096405 (2015)

36. R. Jinnouchi, J. Lahnsteiner, F. Karsai, G. Kresse, M. Bokdam, Phys. Rev. Lett. 122, 225701 (2019)

37. J. Wang, S. Olsson, C. Wehmeyer, A. Pérez, N.E. Charron, G. de Fabritiis, F. Noé, C. Clementi, ACS Central Science 5, 755 (2019)

38. W. Chen, A.L. Ferguson, J. Comp. Chem. 39, 2079 (2018)

39. F. Noé, S. Olsson, J. Köhler, H. Wu, Science 365(6457), $1001(2019)$

40. P. Wirnsberger, A. J. Ballard, G. Papamakarios, S. Abercrombie, S. Racanière, A. Pritzel, D. JimenezRezende, C. Blundell (2020) J. Chem. Phys. 153:144112

41. G. M. Rotskoff, E. Vanden-Eijnden, Trainability and accuracy of neural networks: an interacting particle system approach. (2019). arXiv:1805.00915

42. C. Dellago, P.G. Bolhuis, Transition path sampling and other advanced simulation techniques for rare events, in Advanced Computer Simulation Approaches for Soft Matter Sciences III. ed. by C. Holm, K. Kremer (Springer, Berlin Heidelberg, Berlin, Heidelberg, 2009), pp. 167-233

43. G. Torrie, J. Valleau, J. Comp. Phys. 23, 187 (1977)

44. A. Laio, M. Parrinello, Proc. Natl. Acad. Sci USA 99, $12562(2002)$

45. F. Wang and D. P. Landau, Phys. Rev. Lett. 86, 2050 (2001)

46. C. Dellago, P.G. Bolhuis, P.L. Geissler, Transition path sampling, in Adv. Chem. Phys., Chap. 1. (Wiley, 2002), pp. $1-78$

47. P. G. Bolhuis and D. W. H. Swenson, Adv. Theor. Sim. 4, 2000237 (2021)

48. R.J. Allen, D. Frenkel, P.R. ten Wolde, J. Chem. Phys. 124, 194111 (2005)

49. S. Hussain, A. Haji-Akbari, J. Chem. Phys. 152, 060901 (2020)

50. R. Elber, Annu. Rev. Biophys. 49, 69 (2020)

51. E. Vanden-Eijnden, Transition path theory, in: Computer Simulations in Condensed Matter Systems: From Materials to Chemical Biology, vol. 1, ed. by M. Ferrario, G. Ciccotti, K. Binder (Springer, Berlin, 2006), pp. $453-493$

52. B. E. Husic and V. S. Pande, J. Am. Chem. Soc. 140, $2386(2018)$

53. B.D. Todd, P.J. Daivis, Nonequilibrium Molecular Dynamics: Theory, Algorithms and Applications (Cambridge University Press, 2017)

54. M. S. Green, J. Chem. Phys. 22, 398 (1954)

55. R. Kubo, J. Phys. Soc. Japan 12, 570 (1957)

56. A. Würger, Rep. Prog. Phys. 73, 126 (2010)

57. U. Seifert, Rep. Prog. Phys. 75, 126001 (2012)

58. H. Touchette, Physics Reports 478, 1 (2009)

59. M. Sprik and M. L. Klein, J. Chem. Phys. 89, 7556 (1988)

60. L. Maragliano and E. V. den Eijnden, Chem. Phys. Lett. 426, 168 (2006)
61. D. Frenkel and B. Smit, Understanding Molecular Simulation (Academic Press, London, 2002)

62. D. Marx, J. Hutter, Ab Initio Molecular Dynamics (Cambridge University Press, 2009)

63. D. M. Ceperley, Rev. Mod. Phys. 67, 279 (1995)

64. H. D. Raedt, in MD and MC of Condensed Matter Systems, edited by G. Ciccotti and K. Binder SIF, Bologna, 1996, p. 401

65. D.M. Ceperley, in $M D$ and $M C$ of Condensed Matter Systems. ed. by G. Ciccotti, K. Binder (SIF, Bologna, 1996), p. 443

66. W.H. Miller, in Classical and Quantum Dynamics in Condensed Phase Simulations. ed. by G. Ciccotti, B.J. Berne, D.F. Cocker (World Scientific, Singapore, 1998), p. 617

67. J.C. Tully, in Classical and Quantum Dynamics in Condensed Phase Simulations. ed. by G. Ciccotti, B.J. Berne, D.F. Cocker (World Scientific, Singapore, 1998), p. 489

68. S. Bonella, D. F. Coker, D. MacKernan, R. Kapral, and G. Ciccotti, in Energy Transfer Dynamics in Biomaterial Systems, edited by D. A. M. I. Burghardt, V. May and E. R. Bittner. Springer, Berlin, 2009, p. 415

69. F. Agostini, S. Caprara, and G. Ciccotti, EuroPhys. Lett. 78, 30001 (2007)

70. P. Buijsman, P.G. Bolhuis, J. Chem. Phys. 152, 044108 (2020)

71. L. Zanovello, M. Caraglio, T. Franosch, P. Faccioli, Phys. Rev. Lett. 126, 018001 (2021)

72. J. Behler, G. Csányi, Eur. Phys. J. B 94, 142 (2021). doi: 10.1140/epjb/s10051-021-00156-1

73. J. Köfinger, G. Hummer, Eur. Phys. J. B 94, 245 (2021). doi: 10.1140/epjb/s10051-021-00234-4

74. M. Chen, Eur. Phys. J. B 94, 211 (2021). doi: 10.1140/epjb/s10051-021-00220-w

75. J. Rogal, Eur. Phys. J. B 94, 223 (2021). doi: 10.1140/epjb/s10051-021-00233-5

76. A. Bittracher, J. Moschner, B. Koksch, R. Netz, C. Schütte, Eur. Phys. J. B 94, 195 (2021). doi: 10.1140/epjb/s10051-021-00200-0

77. L. B. Pártay, G. Csányi, N. Bernstein, Eur. Phys. J. B 94, 159 (2021). doi: 10.1140/epjb/s10051-021-00172-1

78. P. G. Bolhuis, Z. Faidon Brotzakis, M. Vendruscolo, Eur. Phys. J. B 94, 188 (2021). https://doi.org/10. 1140/epjb/s10051-021-00154-3

79. S. Ramírez-Hinestrosa, D. Frenkel, Eur. Phys. J. B 94, 199 (2021). https://doi.org/10.1140/epjb/ s10051-021-00209-5

80. F. Grasselli, S. Baroni, Eur. Phys. J. B 94, 160 (2021). https://doi.org/10.1140/epjb/s10051-021-00152-5

81. D. T. Limmer, C. Y. Gao, A. R. Poggioli, Eur. Phys. J. B 94, 145 (2021). doi: 10.1140/epjb/s10051-021-00164-1

82. D. D. Girardier, A. Coretti, G. Ciccotti, S. Bonella, Eur. Phys. J. B 94, 158 (2021). doi: 10.1140/epjb/s10051021-00165-0

83. A. M. N. Niklasson, Eur. Phys. J. B 94, 164 (2021). doi: 10.1140/epjb/s10051-021-00151-6

84. R. D. Skeel, C. Hartmann, Eur. Phys. J. B 94, 178 (2021). doi: 10.1140/epjb/s10051-021-00182-z

85. C. R. A. Abreu, M. E. Tuckerman, Eur. Phys. J. B 94, 231 (2021). doi: 10.1140/epjb/s10051-021-00226-4

86. F. Agostini, E. K. U. Gross, Eur. Phys. J. B 94, 179 (2021). doi: 10.1140/epjb/s10051-021-00171-2 
87. S. C. Althorpe, Eur. Phys. J. B 94, 155 (2021). doi: 10.1140/epjb/s10051-021-00155-2

88. T. Thomas, B. Roux, Eur. Phys. J. B 94, 203 (2021). doi: 10.1140/epjb/s10051-021-00207-7

89. A. S. F. Oliveira, G. Ciccotti, S. Haider, A. J. Mulholland, Eur. Phys. J. B 94, 144 (2021). doi: 10.1140/epjb/s10051-021-00157-0

90. P. Zhou, S. C. Glotzer, Eur. Phys. J. B 94, 243 (2021). doi: 10.1140/epjb/s10051-021-00250-4

91. R. Cortes-Huerto, M. Praprotnik, K. Kremer, L. Delle Site, Eur. Phys. J. B 94, 189 (2021). https://doi.org/10.1140/epjb/s10051-021-00193-w
92. K. M. Kidder, R. J. Szukalo, W. G. Noid, Eur. Phys. J. B 94, 153 (2021). doi: 10.1140/epjb/s10051-021-00153-4

93. R. Menichetti, M. Giulini, R. Potestio, Eur. Phys. J. B 94, 204 (2021). doi: 10.1140/epjb/s10051-021-00205-9

94. A. Giacomello, C.M. Casciola, Y. Grosu, S. Meloni, Eur. Phys. J. B 94, 163 (2021). https://doi.org/10.1140/ epjb/s10051-021-00170-3

95. M. Bernetti, G. Bussi, Eur. Phys. J. B 94, 180 (2021). doi: 10.1140/epjb/s10051-021-00186-9 